\title{
GENETIC VARIATION ASSOCIATED WITH LOW-DENSITY LIPOPROTEIN CHOLESTEROL LEVELS INFLUENCES ZFHX3 EXPRESSION
}

R Martin, M S Koref, A Owens, B Keavney Newcastle University

doi:10.1136/heartjnl-2013-304019.180

The ZFHX3 gene in chromosome 16q22 codes for a transcription factor which controls expression of platelet-derived growth factor receptor beta (PDGRB). Genome-wide association studies (GWAS) have identified several single-nucleotide polymorphisms (SNPs) in this region which are associated with cardiovascular disease phenotypes. rs2106261 and rs7193343 in intron 1 are associated withatrial fibrillation (AF), rs7199343 in intron 1 is associated with Kawasaki disease and rs16971384 in intron is associated with lowdensity lipoprotein cholesterol (LDL-C) levels.

We investigated these associations by genotyping multiple SNPs in order to tag variation in the region of interest. We then looked for associations between SNP genotype and relative expression of the two alleles within an individual, identifying allelic expression quantitative loci (aeQTLs) acting proximally (in cis). This allelic expression approach controls for external influences on gene expression, as these would be expected to act on both alleles equally and so not affect the allelic expression ratio.

Method Whole blood was collected for DNA and RNA extractio from 451 patients at the time of cardiac surgery or cardiac catheterisation. Genotyping was performed on the Sequenom MassARRAY iPLEX Gold platform. Relative allelic expression was determined using the same platform by calculating the ratio of peak areas for each allele of a transcribed SNP, rs740178. Associations between each SNP genotype and the allelic expression ratio were tested using a likelihood maximisation algorithm to phase genotypes and estimate the effect size of the SNP on gene expression. Associations are summarised as p-values.

Results 145 individuals were heterozygous at rs740178. After quality control procedures, allelic expression ratios were determined

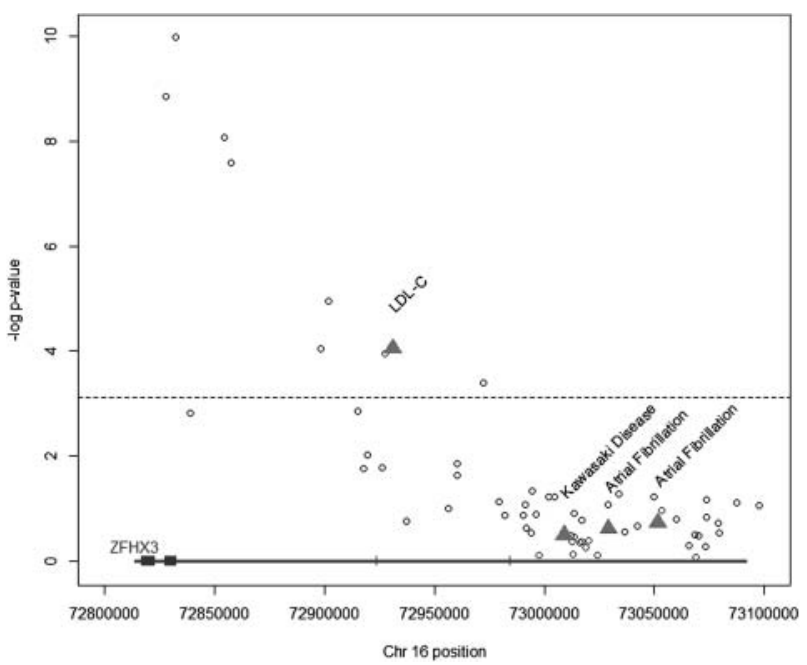

Figure 1 Log p-value by chromosomal position. SNPs identified in GWAS studies are marked with red triangles. The significance threshold is marked with a dashed horizontal line. 

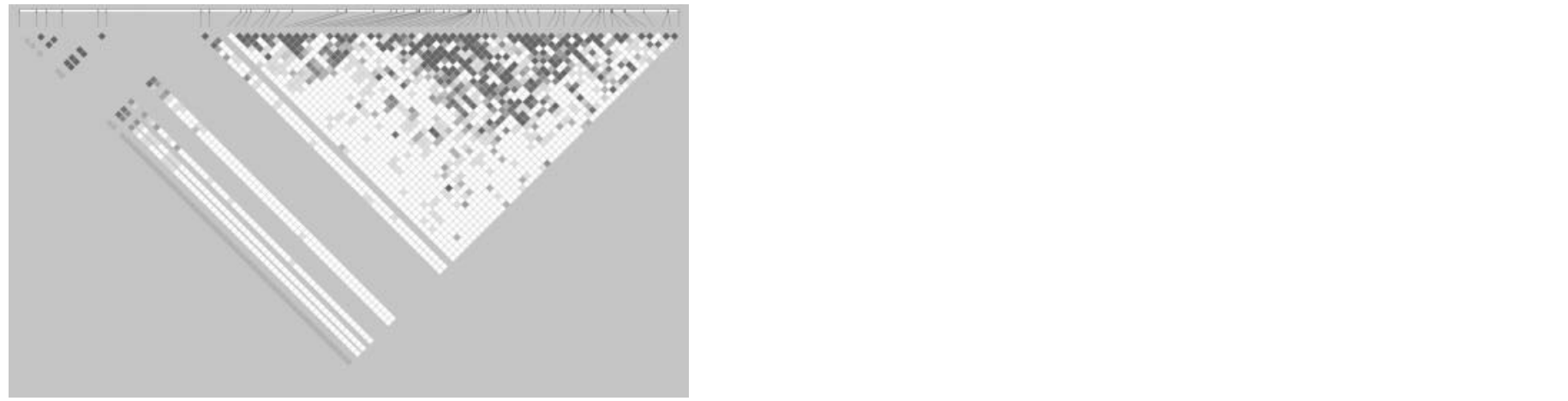

Figure 2 Linkage disequilibrium in the ZFHX3 region. Increasing depth of colour indicates stronger LD.

for 127 individuals. After controlling for multiple association testing 9 SNPs were significantly associated with ZFHX3 expression. The strongest association was seen in the transcribed SNP, rs 740178 ( $p=1.05 \mathrm{E}-10)$ and in SNPs in strong linkage disequilibrium (LD). Associations were also seen with the LDL-C GWAS hit SNP, rs $16971384(p=8.91 \mathrm{E}-05)$ and rs $8055870(p=8.02 \mathrm{E}-04)$, which are not in stong $L D$ with rs 740178. Associations were not seen between ZFHX3 expression and SNPs associated with AF or Kawasaki disease.

Conclusion We have mapped cis-regulation of gene expression in peripheral blood cells at a locus associated with multiple cardiovascular disease phenotypes. SNPs associated with LDL-C levels are significantly associated with ZFHX3 expression. Associations were not demonstrated with SNPs associated with other disease phenotypes; this may be accounted for by tissue specificity of aeQTLs. 